\title{
A New Scientific Paradigm of Reverse Vaccinology: An Annotation
}

\author{
Abu S. M. Giasuddin ${ }^{1, *}$, Khadija A. Jhuma ${ }^{1}$, Waziul A. Chowdhury ${ }^{1}$, Rubayat S. Giasuddin ${ }^{2}$ \\ ${ }^{1}$ Impulse Hospital, ImHS\&RCLtd, 304/E Tejgaon Industrial Area, Dhaka-1208, Bangladesh. \\ ${ }^{2}$ Department of Medicine, Anwer Khan Modern Medical College, Road No. 8A, Dhanmondi, Dhaka-1207, Bangladesh.
}

How to cite this paper: Abu S. M. Giasuddin, Khadija A. Jhuma, Waziul A. Chowdhury, Rubayat S. Giasuddin. (2021) A New Scientific Paradigm of Reverse Vaccinology: An Annotation. International Journal of Clinical and Experimental Medicine Research, 5(4), 431-434. DOI: $10.26855 / \mathrm{ijcemr} .2021 .10 .002$

Received: July 5, 2021

Accepted: July 30, 2021

Published: August 27, 2021

*Corresponding author: Abu S. M. Giasuddin, Impulse Hospital, ImHS\&RCLtd, 304/E Tejgaon Industrial Area, Dhaka-1208, Bangladesh. Email: asmgias@hotmail.com

\begin{abstract}
Since the pioneer work in 1796 by Edward Jenner, Father of Vaccination, many vaccines against infectious diseases have become routinely available saving lives in the community all over the world. The modern terminology of 'Vaccination' was derived from 'Vacca' meaning cow. Renowned biomedical scientists contributed in the development of 'Immunology' as an important biomedical science. Many of them have received 'Nobel Prize' for their noble discoveries in Immunology. The development of nucleic acid sequencing technology has changed the landscape of the evolving field of vaccinology since 1990s. The methodology of Reverse Vaccinology, application of genomic technologies in vaccine research, represented a major revolution in the process of discovering novel vaccines. Bringing a new vaccine from basic research to a product ready for commercial and community use is a very challenging task that requires formulating completely new scientific paradigm. Reverse Vaccinology is a good and classical example of such a paradigm. In fact, it is having, and will have, tremendous applications and implications in the development of protective vaccines particularly against the pandemic 'COVID-19' caused by 'SARS-CoV-2' creating havoc all over the world presently.
\end{abstract}

\section{Keywords}

Vaccine, Vaccination, Vaccinology, Reverse Vaccinology

Vaccines have been traditionally developed empirically by isolating, inactivating and injecting the microorganisms or portions of them that cause disease. The history of vaccinology started in 1798 when Edward Jenner, a county doctor in Gloucestershire, England, UK, observed and discovered that milk maids who suffered from cowpox were secure from, and immune to the future infection of smallpox. He reported 16 cases of resistance to smallpox in farm workers who had recovered from cowpox. In fact, on May 14, 1796, Jenner deliberately inserted 'matter taken from a sore (cowpox) on the hand of a dairymaid into the arm of a 8-year old boy by means of two superficial incisions, barely penetrating the cutis, each about half an inch long'. Two months later, Jenner inoculated the same 8-year-old boy with matter from a smallpox patient, termed the process as 'Variolation'. However, the boy did not develop smallpox, rather developed a small sore at the site of inoculation. The exposure to the mild disease 'cowpox' had made the boy resistant (immune) to the deadly disease 'smallpox'. The modern terminology of 'Vaccination' is thus derived from 'Vacca' meaning 'Cow'. Subsequently, many investigators and scientists such as Louise Pastur, Elic Metchnikoff, Robert Kock, Von Vehring, Jules Bordet, Paul Erlich, Karl Landsteiner, Astrid Fargraeus, Neil Jerne, Macfarlane Burnet, Peter Medawar, Rodney Porter, Ferald Edelman, Henry Claman, Peter Doherty, Rolf Zinkernagal, Cesar Milstein, Georges Kohler, Susumu Tonegawa and many others contributed in the 
development of 'Immunology' as an important biomedical science. Many of these biomedical scientists have received Nobel Prize in different years for their noble discoveries and contributions in the field of Immunology [1, 2, 3].

The application of this practice has essentially remained unchanged for nearly two centuries. The preferred method for vaccine target identification had been the analysis of sera from infected individuals who are protected from reinfection. This procedure fails to identify those components that are not highly immunogenic during infection, but are able to confer protective immunity such as tetanus toxoid [2, 4]. Since the late 1990s, the development of nucleic acid sequencing technologies has changed the landscape of the evolving field of vaccinology. When the genome of the first living organism (Haemophilus influenza, MC 58 strain) was sequenced in 1955, it was realized that genomic technologies, may help to determine the whole proteomic potential of the infectious organism and this will boost the chances of identifying the protein or mixture of proteins that could be used to develop as efficacious vaccine $[5,6]$. This methodology of 'Reverse Vaccinology', the first application of genomic technologies in vaccine research, represented a major revolution in the process of discovering novel vaccines. The term 'Reverse Vaccinology' originates from the change in perspective allowed by the advancements in sequencing technologies $[4,7,8]$.

It allowed the identification of potential vaccine targets in organisms that were difficult to cultivate in the Laboratory. Once all the genes encoded in the genome of a pathogenic species are known, the list of vaccine candidates is finite and, in principle, can be tested in animal models. Therefore, protective antigens would not be missed although it might take time and effort to find them and to define the most effective vaccine formulation $[9,10,12]$. The testing of vaccine candidates in animal models still produces the major bottleneck of the entire 'Reverse Vaccinology' process. There has been a general technological advancement in the software tools that are used throughout the selection process, and in many cases their predictions can now be tested using new experimental methods. Thus, 'Reverse Vaccinology' is an improvement on vaccinology that employs bioinformatics, pioneered by Rano Rappouli and first used against Serotype B Meningococcus and since then, it has been used on several other bacterial vaccines $[4,10,13]$.

A streptococcus and B streptococcus were two of first Reverse Vaccines created, but those bacterial strains induce antibodies that react with human antigens. Therefore, the vaccines for those bacteria needed not to contain homologies to proteins encoded in the human genome in order to not cause adverse reactions or autoimmune diseases. Thus, it has established the need for genome-based 'Reverse Vaccinology' which was later used to develop vaccines for antibiotic-resistant Staphylococcus aureus and Streptococcus pneumonia [4, 11]. It is much more efficient than traditional vaccinology, which requires growing large amounts of specific microorganisms as well as extensive wet laboratory test. However, the downside is that only proteins can be targeted using 'Reverse Vaccinology', whereas conventional vaccinology approaches can find other bimolecular targets such as polysaccharides or lipopolysaccharides $[4,13]$.

The major discoveries that were made in the context of 'Reverse Vaccinology' projects, major milestones in the evolution of reverse vaccinology, are as the following:1995: First complete genome sequence of a living organism; 2000: First application of whole genome sequencing in vaccine research: Formulation of the reverse vaccinology approach; 2002: First application of DNA microarray technology to antigen discovery; 2005: First comparative genomic study of multiple isolate of the same bacterial species and formulation of the pan-genome concept; 2005: First rational design of multi-component protein vaccine for GBS based on the analysis and screening of multiple bacterial genomes; 2006: First formulation of a broadly protective vaccine against Meningococcus B [5-7, 13-15].

Reverse Vaccinology has been a revolution in the field of vaccine discovery for infectious diseases, and has been instrumental in advancing vaccines for bacteria that were thought intractable. Further improvements are still possible that will widen the scope of reverse vaccinology such as the chances of success and speed in the development of vaccines, particularly for complex viruses, such as cytomegalovirus that have a coding capacity, the complexity and sophisticated regulation mechanisms [16]. Computer-aided bioinformatics projects are becoming extremely popular as they help guide the laboratory experiments. Growing number of bioinformatics programmes such as NERVE, VAXIN, PANKPEP, etc. have been making reverse vaccinology information more accessible [11, 17-19].

Bringing a new vaccine from basic research to a product ready for the market through the many stages of development is a challenging task that sometimes requires formulating completely new scientific paradigms [1, 2, 4]. 'Reverse Vaccinology' is a good example of such a paradigm, although many of its original premises have changed. The Reverse Vaccinology approach is, and will continue to be in the future, a fundamental part of vaccine research projects.

Reverse Vaccinology is having, and will have, tremendous applications and implications in the development of protective vaccines for recent Corona Virus Disease 2019 (COVID-19) [20-22]. In fact, bioinformatics approaches 
were employed by researchers to design and introduce a novel multi-epitope vaccine against severe acute respiratory syndrome coronaviraus-2 (SARS-CoV-2) responsible for the pandemic COVID-19. The idea was that potentially it would be able to trigger both $\mathrm{CD}^{4+}$ and $\mathrm{CD}^{8+}$ T-cells inducing protective immune responses against SARS-CoV-2 causative agent for COVID-19 [22-25].

Presently, more than 70 vaccine candidates against SARS-CoV-2 are at some stages of development involving nucleic acid technologies, i.e., reverse vaccinology. The recently approved COVID-19 vaccine developed jointly by PFIZERINC USA and BIONTECH SE GERMANY is a RNA vaccine composed of nucleoside-modified mRNA encoding a mutated form of the spike protein of SARS-CoV-2 and encapsulated in lipid nanoparticles [26-29]. Nevertheless, future investigations of the fundamental mechanisms regulating the human immune responses to pathogens will continue to facilitate the design of new and more efficient vaccines for infectious diseases. Possibly we are entering, rather already entered, into the second era of reverse vaccinology [12, 13, 27-30]. (NB: A comprehensive review on the design and development of COVID-19 vaccines is outside the scope of our present annotation).

\section{Acknowledgements}

The authors would like to appreciate Mr. Mohiuddin Faisal, Computer Programmer, Impulse Hospital, ImHS\&RCLtd, Dhaka-1208, Bangladesh for helping with the references and composing the manuscript.

\section{References}

[1] Giasuddin, A. S. M. (2017). Role of immunologists in the development of health care system. Journal of Immunology and Immunotherapy, 1(1), 2. http//www.imedpub.com/articles.

[2] Greenberg S. A concise history of immunology. www.columbia.edu/itc/hs/medical.../immunology/.../ConcisehistoryImmun ology.pdf. (Retrieved 03 July 2018).

[3] Chappel, H., Haeney, M., Mishbah, S., and Sowden, N. (eds.). (2014). Essential of clinical immunology, $6^{\text {th }}$ edition; Oxford: Wiley Blackwell; 2014. (www.wiley.com/wiley-blackwell).

[4] Donati, C. and Rappuoli, R. (2012). Reverse vaccinology in the $21^{\text {st }}$ century: improvements over the original design. Annals of New York Academy of Sciences, 285, 115-132.

[5] Fleischmann, R. D., Adams, M. D., White, O., Clayton, R. A., Kirkenes, E. F., Kerlavage, A. R., et al. (1995). Whole-genome random sequencing and assembly of Haemophilus influenza Rd. Science, 269, 496-512.

[6] Pizza, M., Scarlato, V., Masignani, Giuliani M. M., Arico, B., Comanducci, M., et al. (2000). Identification of vaccine candidates against serogroup B meningococcus by whole genome sequencing. Science, 287, 1816-1820.

[7] Boerno, S. T., Grimm, C., Lehrach, H., and Schweiger, M. R. (2010). Next-generation sequencing technologies for DNA methylation analyses in cancer genomics. Epigenomics, 2, 199-207.

[8] Nobuta, K., McCormick, K., Nakano, M., Meyers, B. C. (2010). Bioinformatics analysis of small RNA in plants using next generation sequencing technologies. Methods in Molecular Biology, 591, 89-106.

[9] Tettelin, H., Saunders, N. J., Heidelberg, J., Jeffries, A. C., Nelson, K. E., Eisen, J. A., et al. (2000). Complete genome sequence of Neisseria meningitides serogroup B strain MC58. Science, 287, 1809-1815.

[10] Giuliani, M. M., Adu Bobie, J., Comanducci, M., Arico, B., Savino, S., Santini, L., et al. (2006). A universal vaccine for serogroup B Meningococcus. Proceedings of the National Academy of Sciences USA, 103, 10834-10839.

[11] Michalik, M., Djahanshiri, B., Leo, J. C., and Linke, D. (2016). Reverse Vaccinology: The pathway from genomes and epitope predictions to tailored recombinant vaccines. Methods in Molecular Biology, 1403, 87-106.

[12] Rappuoli, R. and Aderem, A. (2011). A 2020 vision for vaccines against HIV, tuberculosis and malaria. Nature, 473,463-466.

[13] Giasuddin, A. S. M., Jhuma, K. A., and Haq, A. M. M. (2018). Reverse vaccinology: Modern challenges in vaccine design and development. Journal of the Medical College for Women and Hospital, 16(1\&2), 23-25.

[14] Tettelin, H., Riley, D., Cattuto, C., and Medini, D. (2008). Comparative genomics: the bacterial pangenome. Current Opinion in Microbiology, 11,472-477.

[15] Berlanda Scorza F., Doro, M., Rodriguez-Ortega, M. J., Stella, M., Liberatori, S., Taddei, A. R., et al. (2008). Proteomics characterization of outer membrane vesicles from the extraintestinal pathogenic Escherichia coli DeltatorIR IHE3034 mutant. Molecular and Cell Proteomics, 7, 473-485.

[16] Stern-Ginossar, N., Weisburd, B., Michalski, A., Li, V. T. K., Hein, M. V., Huang, S.-X., et al. (2012). Decoding human cytomegalovirus. Science, 338, 1088-1093.

[17] Cheng, L., Lu, W., Kulkarni, B., Pejovic, T., Yan, X., Chiang, J.-H., et al. (2010). Analysis of chemotherapy response programs in ovarian cancers by the next-generation sequencing technologies. Gynecology and Oncology, 117, 159-169.

[18] Contreras, M., Villar, M., Artigas-Jerónimo, S., Kornieieva, L., Mytrofanov, S., de la Fuente, J. (2018). A reverse vaccinology 
approach to the identification and characterization of Ctenocephalides felis candidate protective antigens for the control of cat flea infestations. Parasites and Vectors, 11(1), 43. doi: 10.1186/s13071-018-2618-x.

[19] Dalsass, M., Brozzi, A., Medini, D., and Rappuoli, R. (2019). Comparison of Open-Source Reverse Vaccinology Programs for Bacterial Vaccine Antigen Discovery. Frontiers in Immunology, 10, 113. DOI: 10.3389/fimmu. 2019.00113.

[20] Wu, Z. and McGrogen, J. M. (2020). Characteristics of and Important Lessons from the Coronavirus Disease 2019 (COVID-19) Outbreak in China. Journal of American Medical Association, 323(13), 1239-1242. doi:10.1001/jama.2020.2648.

[21] Dayarathana, S., Jeewandara, C., Gomes, L., Somathilaka, G., Jayathilaka, D., Vimalachandran, V., et al. (2020). Similarities and differences between the cytokine storms in acute dengue and COVID-19. Research Square, doi: https://doi.org/10.21203/rs.3.rs-39133/vi.

[22] Gupta, E., Mishra, R. K., and Niraj, R. R. K. (2020). Identification of potential vaccine candidates against SARS-CoV-2, a step forward to fight COVID-19: A reverse vaccinology approach. bioRxiv, DOI: http://doi.org/10.1101/2020.04.13.039198 (Retrieved 16 June 2021).

[23] Enayatkhani, M., Hasaniazad, M., Faezi, S., Gouklani, H., Davoodian, P., Ahmadi, N., et al. (2021). Reverse vaccinology approach to design a novel multi-epitope vaccine candidate against COVID-19: An Insilco study. Journal of Biomolecular Structure and Dynamics, 39(8), 2857-2872. doi: 10.1080/07391102.2020.1756411.

[24] Bansal, A., Padappayil, R. P., Garg, C., Singal, A., Gupta, M., Klein, A. (2020). Utility of artificial intelligence amidst the COVID 19 pandemic: A review. Journal of Medical Systems, 44: 156. Available from: https://doi.org/10.1007 /s10916-020-01617-3.

[25] Chen, W. (2020). Promise and challenges in the development of COVID-19 vaccines. Human Vaccines \& Immunotherapeutics, 16(11), 2604-2608. doi:10.1080/21645515.2020.1787067. Available from: https://doi.org/10.1080/21645515.2020.1787067.

[26] Burton, D. R. and Walker, L. M. (2020). Rational vaccine design in the time of COVID-19. Cell Host \& Microbe, 27 (5), 695-698. Available from: https://doi.org/10.1016/j.chom.2020.04.022.

[27] Wikipedia. COVID-19 vaccine. Available from: https://en.m.wikipedia.org/wiki/COVID 19 Vaccine. (Accessed on 05 December 2020)

[28] www.cepi.net. Newvaccines for a safer world. CEPI 2020. (Retrieved 15 December 2020)

[29] www.cept.net. CEPI’s COVID-19 vaccine portfolio. CEPI 2020 (Retrieved 15 December 2020)

[30] Rappuoli, R., Bottomley, M. J., D’Oro, U., Finco, O., De Gregorio, E. (2016). Reverse vaccinology 2.0: Human immunology instructs vaccine antigen design. Journal of Experimental Medicine, 213(4), 469-481. Available from:www.jem.org/cgi/doi/10.1084/jem.20151960. 